\title{
A Dynamic Slot Allocation Algorithm in High-Band Radio Networks
}

\author{
Shentao Wang ${ }^{1, *}$, Xiaoli Liu ${ }^{1}$, Bin Liü, Shibiao He${ }^{1}$, Shengze Qiang ${ }^{1}$, Rui Li ${ }^{1}$ and Junqiang $\mathrm{He}^{1}$ \\ ${ }^{1}$ Institution of Chongqing Communication, Chongqing 400035, China \\ ${ }^{2}$ PLA 61655, Chongqing 402764, China \\ *Corresponding author
}

\begin{abstract}
The High Band Radio network has extensive value in the military application. A Dynamic Slot Allocation Algorithm (DSA) based on the High Band Radio network TDMA protocol is proposed in this paper, DSA algorithm allocates slots dynamically according to the difference of network traffics. The simulation results show that DSA algorithm can sustain TDMA protocol improving the network characters according to the difference of the network traffics.
\end{abstract}

Keywords-high band radio networks; TDMA protocol; dynamic slot allocation

\section{INTRODUCTION}

High bandwidth network radio can accomplish long distance and high throughput information transmission with directional beam antenna technology. The networking waveform of the radio, based on TDMA protocol, allocates slot dynamically according the traffic requirements, accomplishes the neighbor discovery, and adjusts the data rate according SNR (Signal to Noise Ratio). Wherein slot allocation algorithm is an important part of the network waveform. Slot allocation algorithm can be divided static slot allocation algorithm, dynamic slot allocation algorithm, and the combination algorithm of static and dynamic slot allocation.

Static slot allocation algorithm, Making Slot allocation scheme during network initialization, can reduce the network collision and improve the fairness using the slot. But it also reduces slot utilization rate, limits the improvement of the network throughput. On this basis, dynamic slot allocation algorithm is proposed. This type of algorithm, allocated slot dynamically according traffic, can improve slot utilization. The difficult of this type of algorithm is to make the principle of slot application and slot release. Literature [1-4], adopted centralized coloration algorithm, complete the slot allocation. These algorithms, getting the topology information of two or three hops firstly, increase the cost and complexity of the protocol. The type of dynamic competition protocol [5-7] reserve slots with neighbor node through a simple and reliable handshake mechanism. But this type of protocol, based on slot allocation distributed equally, didn't allocate finite slot according to actual traffic.

A Dynamic Slot Allocation Algorithm (DSA) based on the High Band Radio network TDMA protocol is proposed in this paper, DSA consists of three parts, data rate statistic algorithms, slot release strategy, and slot application strategy. The data rate statistical algorithm accomplishes statistics of data cached in MAC layer. The slot release strategy and slot application strategy accomplish the selection of application or release, and the confirmation of the number of relevant slot. The simulation results show that DSA algorithm allocates slots dynamically according to the difference of network traffics, and can sustain TDMA protocol improving the network characters according to the difference of network traffics.

\section{DYNAMIC SlOt AlLOCATION ALGORITHM}

MS Word Authors: please try to use the paragraph styles contained in this document.

\section{A. Data Rate Statistic Algorithm}

Assuming that there are fifteen queues caching data in MAC layer, each node corresponds to a queue. Define the traffic of each node is $\operatorname{Traffic}_{i}(1 \leq i \leq 15), \quad i$ is the identification of node. There are fifty data slot in the network, the number of slot of node $i$ is Slot $_{i}=\left\{k_{1}, k_{2}, \cdots, k_{n}\right\} \quad\left(\right.$ Slot $\left._{i} \in 50\right)$, and the traffic corresponding to relevant slot is Slot $_{i}=\left\{k_{1}, k_{2}, \cdots, k_{n}\right\} \quad\left(\right.$ Slot $\left._{i} \in 50\right), j$ is the identification of slot. The total traffic of node $i$ is $\sum_{j=1}^{\text {Slot }_{i}}$ Traffic $_{-}$Slot $_{i}$. Due to the traffic may be different in each slot, at every time of slot allocation, assume that the minimum traffic of the new allocated slot is Traffic $_{\text {min }}$. On the basis of the above parameters, make the selection of slot release or slot application.

\section{B. Slot Release Strategy}

If Traffic $_{i} \leq \sum_{j=1}^{\text {Slot }_{i}}$ Traffic $_{-}$Slot $_{j}$, remain the number of slot, and set the frame timer to five, if the five consecutive frames meet the formula, calculate the number of release slot $k$ :

The traffic to release, as follows:

$$
\text { Traffic }_{\text {free }}=\left(\sum_{j=1}^{\text {Slot }_{i}} \text { Trarric }_{-} \text {Slot }_{j}\right)-\text { Traffic }_{i} .
$$

Assume Traffic $_{\max }$ is maximum traffic of node $i$ that is Traffic $_{\text {max }} \geq$ Traffic $_{-}$Slot $_{j}$, Traffic $_{\max } \in\left\{\right.$ Traffic $_{-}$Slot $\left._{j}\right\}$. 
So $k$, the number of slot to release should meet the formulation:

$$
k=\text { Traffic }_{\text {free }} / \text { Traffic }_{\max } .
$$

\section{Slot Application Strategy}

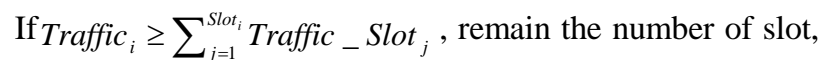
and set the frame timer to five, if the five consecutive frames are meet the formula, calculate the number of application slot $k$.the new increased traffic is

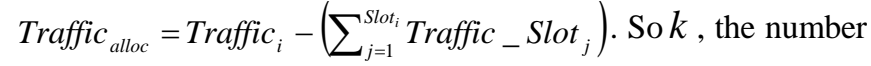
of slot to apply, should meet the formulation $k=\left[\right.$ Traffic $_{\text {alloc }} /$ Traffic $\left._{\min }\right]$.

\section{PROCEDURE OF DSA}

\section{A. Flow of Slot Application}

Step 1: "request node” send slot application packet, including local node ID, destination ID, local geographic information;

Stpep2: “reply node” send slot replication packet, including local node ID, destination ID, local geographic information, and local free slots table;

Step 3: "request node" send slot confirmed packet, including local node ID, destination ID, and mutual free slot;

Step 4: once the "reply node" receive the confirmed packet, update the slot information table.

\section{B. Flow of Slot Release}

Step 1: "request node" send slot release packet, including local node ID, destination ID, local geographic information;

Step 2: "reply node” send slot replication packet, including local node ID, destination ID, local geographic information, and local slot to free;

Step 3: "request node" send confirmed packet, including local node ID, destination ID, and mutual slot to release;

Step 4: once the "reply node" receive the confirmed packet, update the slot information table.

\section{ANALYSIS OF SimUlation}

To validate the performance of DSA, it is compared with static slot algorithm (SSA) based on OPNET network simulation software. The traffic model obeys ON/OFF distribution.

\begin{tabular}{|c|c|}
\hline \multicolumn{2}{|c|}{ TABLE I. SIMULATION PARAMETERS } \\
\hline Paper Size & $21 \mathrm{~cm} \times 29.7 \mathrm{~cm}$ \\
\hline Number of nodes & 15 \\
\hline Network range & $400 \mathrm{~m} \times 400 \mathrm{~m}$ \\
\hline Simulation time & $10 \mathrm{~min}$ \\
\hline Speed of mobility & $0 \sim 10 \mathrm{~m} / \mathrm{s}$ \\
\hline destination of node & $1 \sim 15$ \\
\hline ON duration & exponential $(10.0)$ \\
\hline OFF duration & exponential(5.0) \\
\hline
\end{tabular}

Figure 1 is the chart of data slot utilization comparisons. The formulation of data slot utilization, as follows:

$$
\text { data_slot_utilization }=\frac{\text { the number of data_slot_occupied }}{\text { the number of all network slot }}
$$

Figure 2 is the chart of network throughput comparisons. The formulation of network throughput, as follows:

$$
\text { network_throughput }=\frac{\text { total data received of network }}{\text { the time of network runing }}
$$

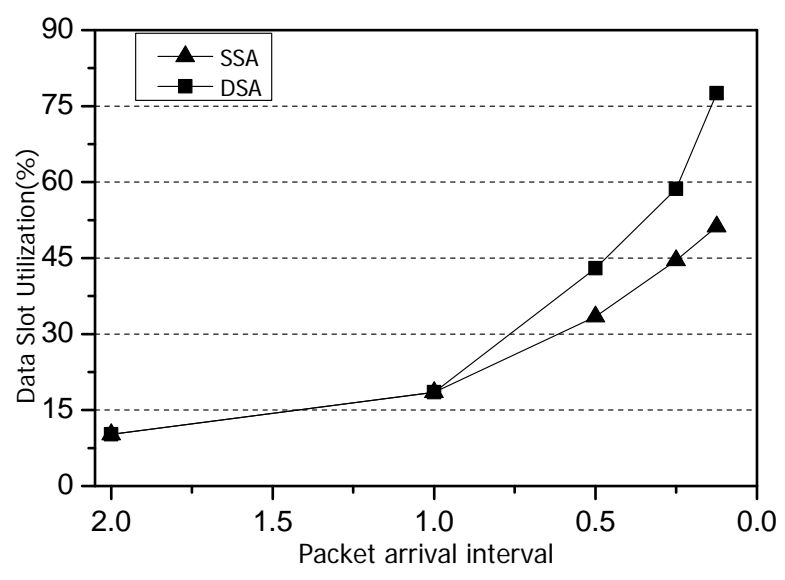

FIGURE I. DATA SLOT UTILIZATION

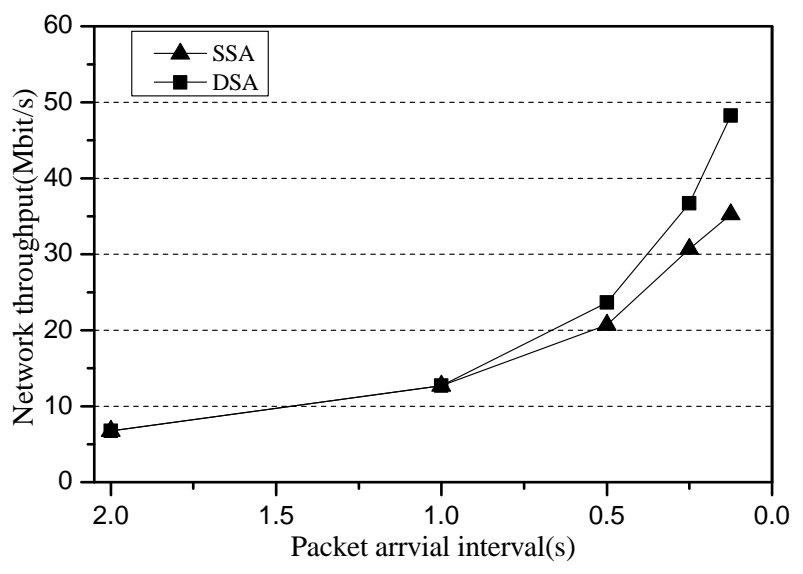

FIGURE II. NETWORK THROUGHPUT

From Figure 1 and Figure 2, it can be seen that when the interval of packet arrival is higher, the data slot utilization and network throughput of SSA is the same as DSA. This is because the slots of node meet the traffic preferably in SSA and DSA. But with the interval of packet arrival lower, due to the slot allocation character of SSA, the data slot utilization and network is lower than DSA. 


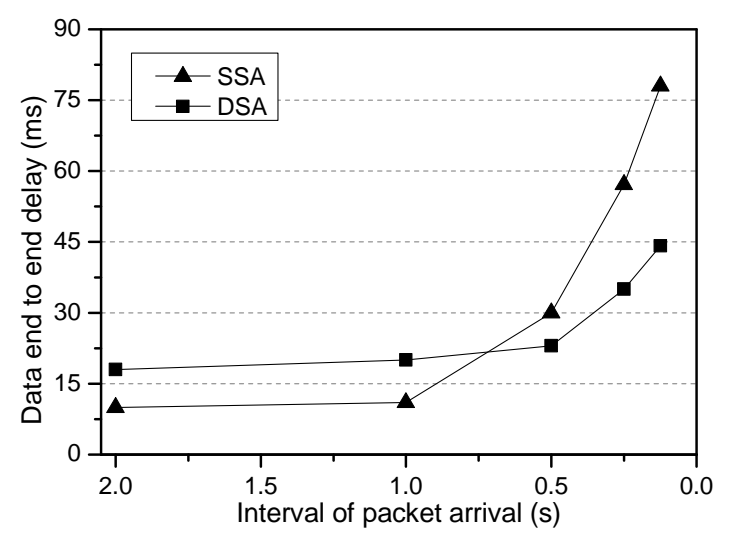

FIGURE III. DATA END TO END DELAY

Figure 3 is the chart of data end to end delay comparisons. The formulation of data end to end delays, as follows:

$$
\text { data end to end delay }=\frac{\text { the total data delay }}{\text { the total number of received data }}
$$

From Figure 3, it can be seen that when the interval of packet arrival is higher, data end to end delay of DSA is higher than SSA, this is because of the dynamic slot allocation character of DSA. Before data transmission, slots application should be completed, but it increases the data end to end delay in DSA. With the interval of packet arrival lower, the slots of node cannot meet the traffic preferably in SSA, the number of data cached in MAC layer queues overstocks, making data end to end delay is more serious. Due to the dynamic slot application and release character of DSA, the increased extent of data end to end delay is lower than SSA.

\section{CONCLUSIONS}

A Dynamic Slot Allocation Algorithm (DSA) based on the High Band Radio network TDMA protocol is proposed in this paper, DSA algorithm allocates slots dynamically according to the difference of network traffics. The simulation results show that DSA algorithm can sustain TDMA protocol improving the network characters according to the difference of the network traffics.

\section{ACKNOWLEDGMENT}

This work is supported by Laboratory capacity improvement program (No: cstc2014pt-sy0010), Chongqing college and universities good science and research translation significant project (No: KJZH14112).

\section{REFERENCES}

[1] MOSCIBRODA T, WATTENHOFER R. Coloring unstructured radio networks[C]. Proceedings of the 17th Annual ACM Symposium on Parallelism in Algorithms and Architectures. New York, USA, 2005:3948.

[2] SRINIVASAN P, RAJIV G. Distributed algorithms for coloring and domination in wireless Ad Hoc networks[C]. Proceedings of FSTTCS04. Chennai, India, 2004:447-459.
[3] HU Zhiyuan, Guo Jianding, WANG Jing, et al. Spatiotemporal channel assignment in multi-radio wireless mesh networks [J]. Journal of Chongqing University, 2011, 34(2):26-31.

[4] ZHANG Zhen-sheng. DTRA: directional transmission and reception algorithms in wlans with directional antennas for QoS support [J]. IEEE Network, 2005, 19(3): 27-32.

[5] SAYADI A, WEHBI B, LAOUITI A. One shot TDMA based reservation MAC protocol for wireless Ad Hoc network[C]. Proceedings of Vehicular Technology Conference. Evry, France, 2011:1-5.

[6] XU Mingxia, ZHAO Minjian, SONG Zhengwei, et al. An on-demand and dynamic slot assignment protocol for Ad Hoc networks[C]. Proceedings of APCC06. Busan, Korea, 2006:1-5.

[7] VALLATI C. Dynamic resources allocation in wireless mesh network[C]. Proceedings of IEEE International Symposium on World of Wireless, Mobile and Multimedia Networks. Pisa, Italy, 2011:1-3. 